SSC09-VI-9

\title{
Rapid Assembly of Spacecraft Structures for Responsive Space
}

\author{
Shazad Sadick, Roopnarine ${ }^{1}$, Irene Yachbes \\ Honeybee Robotics Spacecraft Mechanisms Corporation \\ 460 West $34^{\text {th }}$ Street New York, NY 10001; 646-459-7806 ${ }^{1}$ \\ roopnarine@honeybeerobotics.com ${ }^{1}$
}

\begin{abstract}
In order to achieve a six-day spacecraft architecture, the assembly, integration \& testing (AI\&T) of the Satellite Bus could be drastically reduced by stocking component-ready modular panels for assembly. The assembly of the structure itself, however, needs to be accelerated from the typical process of securing panels with dozens of mixedsize fasteners and the associated verification, tooling, and documentation that must also take into consideration the need to pass electrical and thermal connections across panels of the bus. A method for rapidly providing a stiff mechanical attachment across panels of a spacecraft bus, while simultaneously providing electrical and thermal continuity, helps to further realize the goals of Responsive Space (RS). A fastening strategy has been developed for enabling rapid assembly of a spacecraft bus structure using Honeybee's patented Quick Insertion Nut (QIN) technology. The QINs are embedded in manifolds which reside at each edge inside the spacecraft bus (the manifold includes panel-to-panel electrical interconnects) and together form a skeletal structure for the spacecraft panels. Initial FEA analyses show that a bus construction based on this concept is capable of meeting the natural frequency requirements for a wide array of launch vehicles and that the QINs themselves are capable of withstanding very high tensile loads with positive margins of safety.
\end{abstract}

\section{INTRODUCTION: STRUCTURAL ASSEMBLY \& THE RS TIMELINE}

A goal of the Responsive Space (RS) thrust is to enable turn-around of a tactical satellite within six days, from mission call-up to on-orbit operation. ${ }^{1}$ The ability to produce such a spacecraft would be a vast departure from the status quo of producing large, complex, costly custom spacecraft that require a period of years to deploy. ${ }^{2}$ More recently, RS-type spacecraft have demonstrated timescales on the order of several months to two years. While progress has been made in developing modular small satellites with shorter developmental timescales, every aspect of the spacecraft development process needs to be reassessed in order to achieve the goals of a truly responsive tactical space program to rapidly meet the tactical needs of the war-fighter.

One approach to achieving responsive space may be to have stockpiles of satellites readily available for launch as needed; however, this approach is costly and suffers from the potential for quick obsolescence. Ideally a single modular architecture will be compatible with a range of mission types, orbits and related space environments, launch vehicles, and technology upgrades. ${ }^{3}$
Assembly, integration and test typically account for 6 months to 2 years of the spacecraft production cycle. This process could be drastically reduced by stocking component-ready modular panels for assembly. Even with the pieces of a spacecraft bus and payload prepared for integration, the assembly of the structure itself needs to be sped from the typical process of securing panels with dozens of mixed-size fasteners and the associated verification, tooling, and documentation. Likewise, assembly of the structure also must take into account the need to pass electrical and thermal connections across the panels of the bus. A method for rapidly providing a stiff mechanical attachment across panels of a spacecraft bus while simultaneously providing electrical and thermal continuity would help to further realize the goals of RS. It will also be crucial to demonstrate quick disassembly of bus panels in order to swap out faulty components, accommodate upgrades or support last-minute component changes to satisfy changing mission needs.

An approach to rapid assembly of a bus structure could be even more valuable if it can be proven reliable enough to help eliminate other time-consuming steps in the assembly, integration and test cycle beyond the physical assembly time. 
For example, a single attachment type which can be used to assemble pre-qualified elements of certain structural configurations could be proven to reduce or eliminate:

- Detailed design time

- Coupled loads analysis

- Dynamic testing

- Thermal-vacuum testing

\section{REQUIREMENTS AND BACKGROUND}

Honeybee Robotics Spacecraft Mechanisms Corporation (Honeybee Robotics) has been funded under the Small Business Innovation Research (SBIR) program to develop a structural attachment method to meet the needs of RS. The basic requirements as defined by the SBIR solicitation topic, along with others derived by consultation with industry partners and literature review, were as follows:

The typical RS spacecraft shall have the following characteristics:

- Generalized 3'x 3'x 2' rectangular bus

- Rapid assembly strategy compatible with two configuration representatives (a rectangular bus with internal cruciform and a hexagonal bus potentially with internal panels) of Joint Warfighting Space (JWS) class satellites

- Bus mass is $200 \mathrm{~kg}$

- Bus center of mass is located at its geometric center

- Payload mass is $200 \mathrm{~kg}$ (attached directly to top of bus)

- Payload center of mass of is located 60.96 $\mathrm{cm}$ above top of the bus

The attachments and bus structure must have sufficient strength and rigidity to be capable of surviving the ground, flight and orbital loads associated with the Minotaur I launch vehicle. These include steady state acceleration, acoustic impingement, random vibration and pyroshock. ${ }^{4}$ Other appropriate launch vehicle interfaces may include Minotaur IV and the Evolved Expendable Launch Vehicle (EELV) Secondary Payload Adapter. Microsats are driven by stiffness issues, rather than strength. Strength verification is generally not needed. Many of these satellites are designed with additional margin for certain loading scenarios. This extra margin allows many of these satellites to avoid static loads testing. ${ }^{5}$ Acoustic testing is also generally unnecessary for microsats. The most important consideration is fundamental frequency. Some expected lower limits are ${ }^{6}$ :

- $\quad$ ESPA (EELV Secondary Payload Adapter): 35 $\mathrm{Hz}$

- Minotaur: $12 \mathrm{~Hz}$ (including effects of an isolation system)

- $\quad$ SpaceX Falcon-1: $\sim 25 \mathrm{~Hz}$

Designing to $50 \mathrm{~Hz}$ covers all the above launch vehicle scenarios, providing added flexibility, and also simplifies coupled loads analysis (CLA) or may even eliminate the need for a unique CLA for each satellite build. ${ }^{6}$ Therefore, by designing our attachment strategy accordingly, not only will we be reducing assembly time but we will also save time in spacecraft design and analysis. While imposing this stiffness requirement could mean that the fastening hardware will be bulkier, structural considerations are nonetheless relatively more important than mass. As long as the fastening strategy does not introduce such a significant mass increase that it changes the satellite class, focus on lightening is generally not a requirement.

The assembly of the bus with all components shall be compatible with an 8-hour overall satellite build process. The portion allotted to assembly of the structure alone shall be half an hour. This assembly process shall enable accurate and stiff connection of structural members with minimal infrastructure (i.e. cost and complexity of required tooling and fixturing and training of assembly technicians). The attachment shall also enable rapid disassembly of panels to swap out components for last minute configuration changes or to replace components rather than undertake lengthy troubleshooting. The assembly process shall also induce minimal misalignment for purposes of accurate spacecraft pointing.

We can consider the following scenario as an example. In the typical assembly process, small spacecraft integration takes place over six months and assembly traditionally requires four to six weeks. The base plate is bolted onto a flat GSE fixture and side panels are assembled onto the base plate. The panels are assembled with $160 \mathrm{ksi}$ fasteners in various sizes (\#10 size or larger) and bosses with helicoil inserts spaced every two to three inches along the edge of the panel. About half a day is required to torque, tighten down and stake one side panel to the base plate with a couple of hours alone to tighten down 25 bolts. Documentation of the assembly is perhaps the most time-intensive step of the process.

In contrast, the envisioned responsive spacecraft will allow for stocked panels with integrated components to be 
taken off the shelf and rapidly assembled. A structural attachment that provides electrical and thermal continuity between panels would enable true modularity, avoiding the steps of harness and thermal control routing across panels. An integrated electrical connection should conform to Space Plug and Play Avionics (SPA) standards currently being developed $^{7}$.

A thermal connection could take many forms, dependent on the design of the spacecraft ${ }^{8}$. While it is possible that each panel could potentially be thermally self-sufficient, this could vary widely based on orbit profile, component layout and panel construction and interfaces. A simple scenario could use a dry fit connection across panels with appropriate surface finish (of 63 micro inches or less). A preload on the attachment in the range of hundreds of psi would also assure heat transfer across the joint. For complex thermal scenarios, a heat pipe across a radiator to spread heat could be terminated to header blocks at the joint and, if necessary, conducted to adjacent panels.

\section{$\begin{array}{lll}\text { CONCEPT } & \text { AND } & \text { PROTOTYPE }\end{array}$ DEVELOPMENT}

\section{Quick Insertion Nut (QIN)}

Honeybee Robotics has developed a fastening strategy for enabling rapid assembly of a spacecraft bus structure, which is uniquely enabled by the use of our patented Quick Insertion Nut (QIN) ${ }^{9}$ technology. The QIN (shown in Figure 1) was originally developed for compatibility with astronaut extravehicular activity tasks (where reduced dexterity is problematic), for robotic assembly tasks, and for fasteners requiring high vibration resistance such as experienced in the launch environment. With this fastening strategy, a standard bolt can be rapidly pushed into the QIN and then, with about one turn, the joint can be preloaded without significant support equipment or operator skill. Detail of the operation sequence of the QIN is shown in Figure 2.

These QINs are embedded in manifolds which reside at each edge and corner inside the spacecraft bus that together comprise a skeleton for the spacecraft panels. This attachment strategy provides structural integrity in tension, compression, shear and torsion and the skeletal frame increases the fundamental frequency of the entire structure. The manifolds at the corners (vertical manifolds) permit side panel-topanel attachment while the manifolds along the top and bottom (horizontal manifolds) permit side panel to top and bottom plate attachments. The manifold includes panel-to-panel electrical interconnects which would interface with panel integrated electronics such as used in Plug-n-Play Sat. When the panels are assembled to the manifolds, a robust structural, electrical, and thermal connection for the bus is achieved (it is also possible that thermal fluid loop connections can be simultaneously made as the panels are connected). The manifold frame structure, along with a complete satellite bus assembly and assembly sequence are shown in Figure 3 and Figure 4. The manifolds serve as structural beams and provide good contact at the interfaces between panels to enable thermal transfer by conduction (thermal conductance is increased due to the increased surface area of contact between the panels and the manifolds) and thereby enhance heat dissipation and distribution and reduce thermal gradients across the satellite.

The angled shape of the vertical manifold determines the shape of the satellite. A 90-degree angled manifold creates a square or rectangular shaped satellite, while a 120-degree angled manifold creates a hexagonal shaped satellite. Incidentally, if stacking architectures were envisioned for future spacecraft, the skeletal manifolds attachment strategy would also lend itself to assembly of multiple component decks within the bus.

We also envision that this attachment strategy may be used for assembling components to spacecraft panels as well, although this may be done with direct componentto-panel connections with the QINs rather than with a manifold in between. This will be investigated in future work.

An alternate approach to the use of a manifold would be to have the QINs along each panel edge and to fasten panels directly to one another. While the use of the manifolds will increase the overall mass of the satellite (clearly a modular bus architecture will require trades where not all aspects of the design will be optimized), the advantages already discussed make it a more desirable approach. Preliminary FEA studies show natural frequency with this method is above $50 \mathrm{~Hz}$.

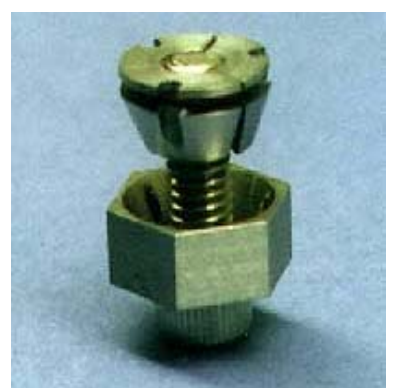

Figure 1: Partial Disassembled View of Quick Insertion Nut. 

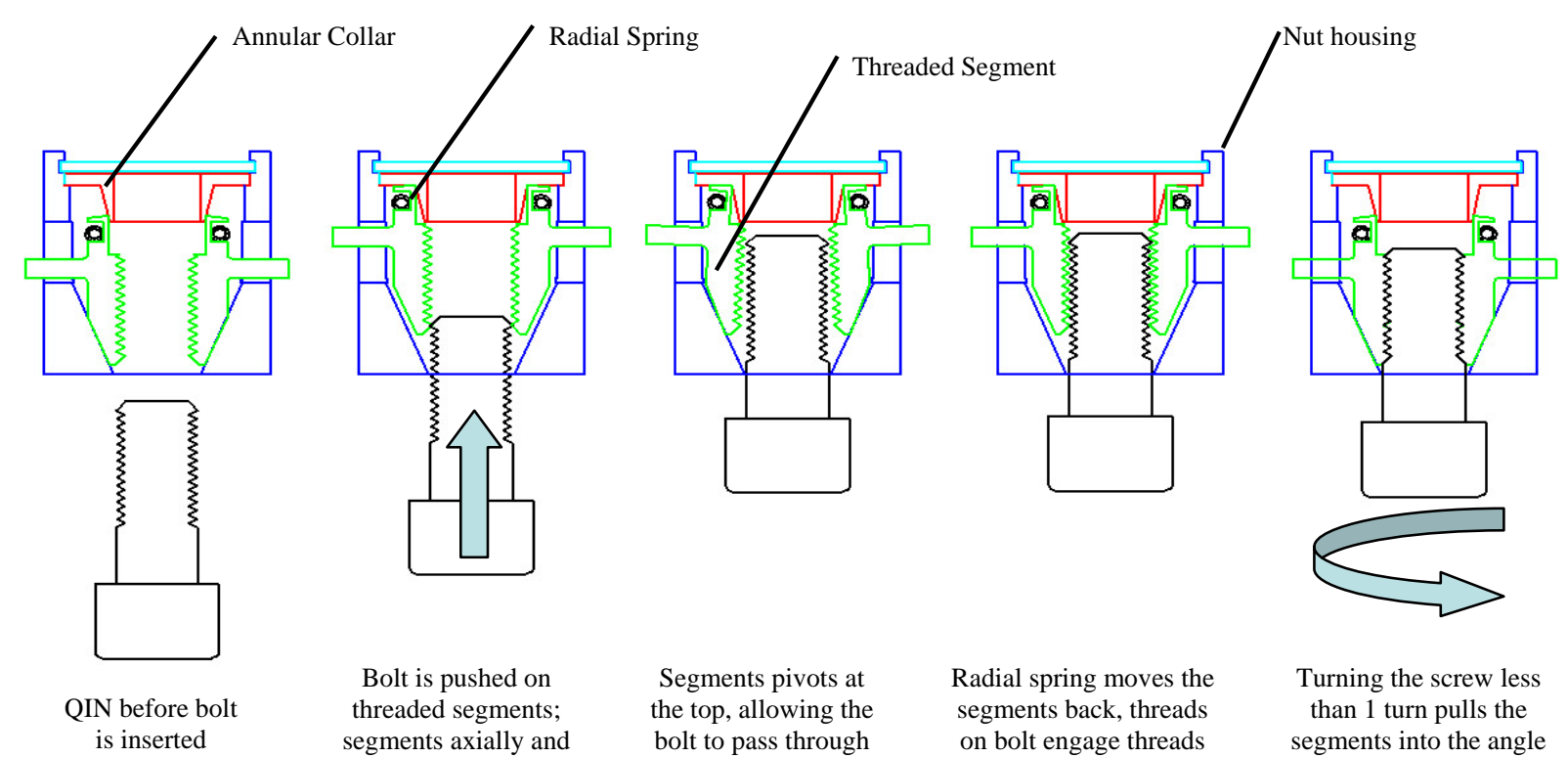

Bolt is pushed on threaded segments; segments axially and radially

Segments pivots at the top, allowing the bolt to pass through the threaded segments
Radial spring moves the segments back, threads on bolt engage threads on the segments
Turning the screw less than 1 turn pulls the segments into the angle housing of the nut

Figure 2: Cross sectional views showing the QIN sequence of operation.
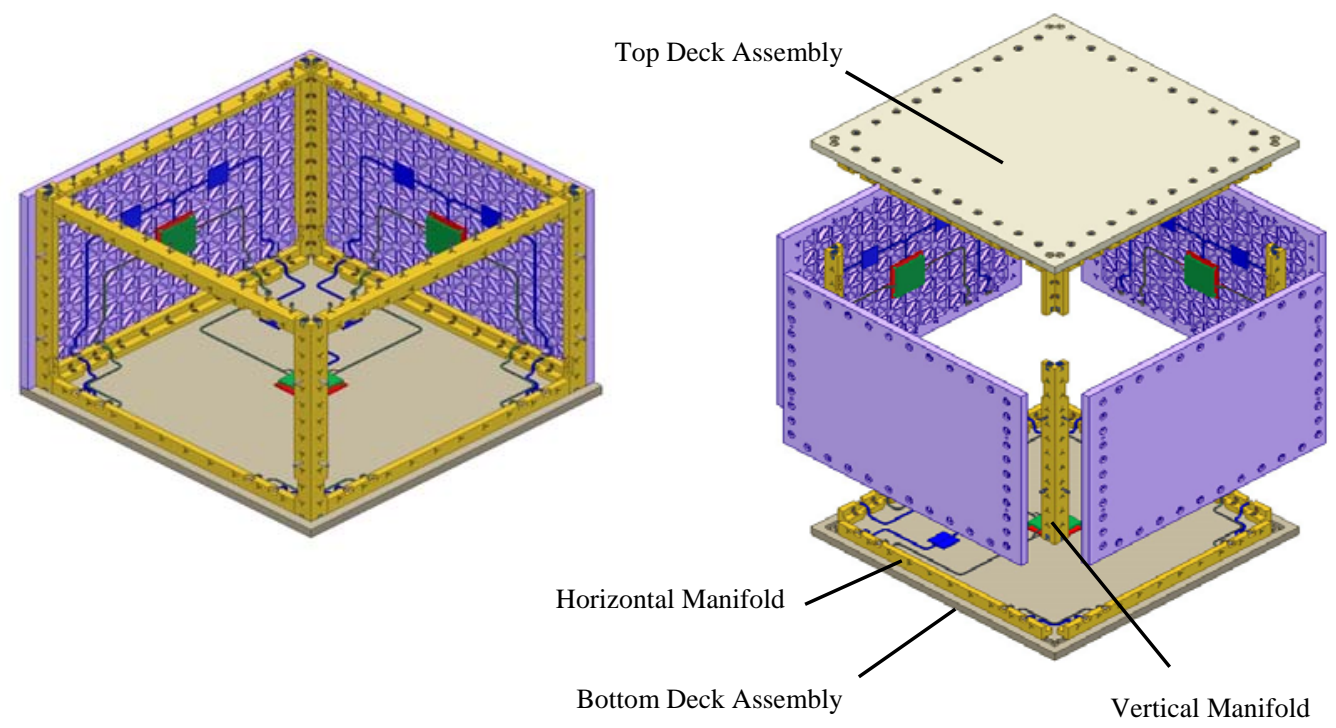

Figure 3: Satellite bus structure and electrical connections using Honeybee's rapid spacecraft assembly solution 


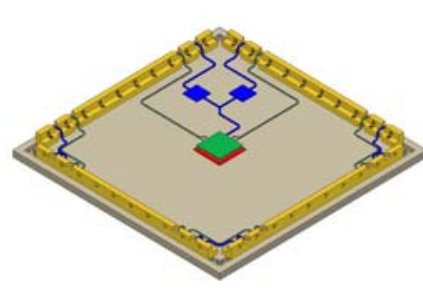

Start with a pre-assembled bottom deck assembly

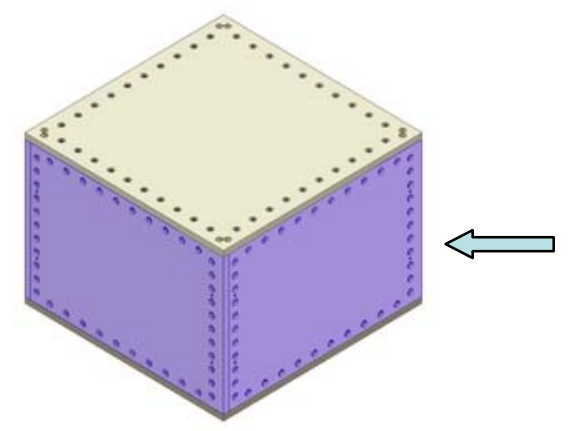

Attach last side panel; Test/inspect satellite's functionalities; after satellite checks out, torque all fasteners down

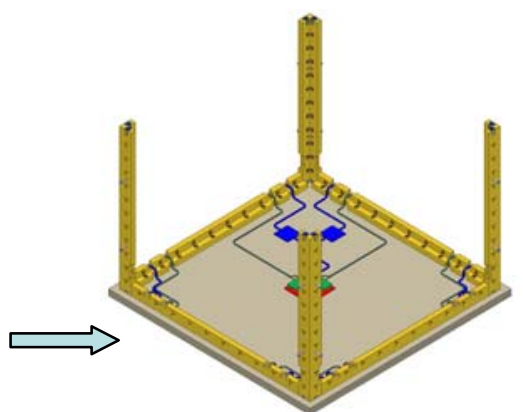

Attach vertical manifolds

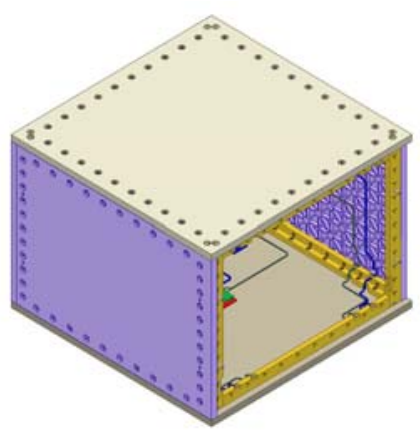

Attach pre-assembled top deck assembly

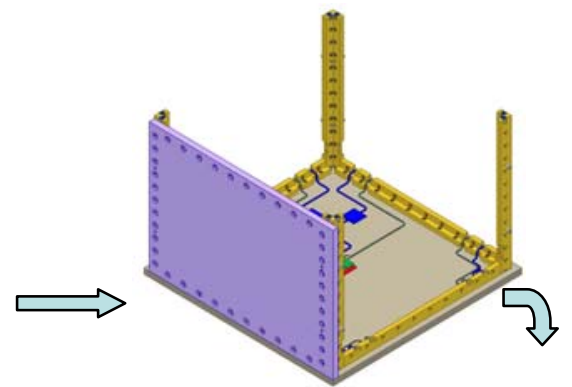

Attach side panel onto manifold, push in fasteners until head "bottoms out" on panel

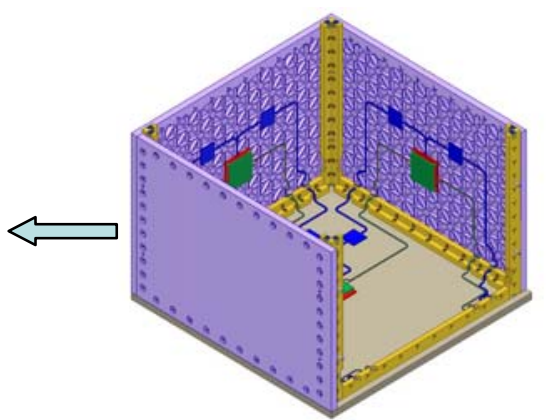

Attach two additional side panels onto manifolds

Figure 4: Assembly sequence of a square satellite bus.

The QIN aids in the rapid assembly of satellites by decreasing the time needed to install a fastener onto the structure. For one fastener, this time saving may not seem like a lot, but considering that each satellite panel can contain as many as 40-50 fasteners, the total time saving can be advantageous in rapid satellite production. Shown in Table 1 is a comparison of the mating sequences between a standard nut and a QIN.

The fastening operation itself is very quick and since the components are already embedded, the time typically required to find and place fasteners is cut out. Because identical fasteners will be used, only one tool, torque value, and verification process will be required. In the case of a simple thermal design, the same preload verification used for the structural connection would serve doubly as the thermal verification. Rapid disassembly of the panels from an integrated structure using standard tooling may be accomplished by removing preload and using the quick-removal feature on the patented system.

The alignment of the connectors on the panels and the horizontal manifolds are critical during satellite assembly. Tapered holes (on the panels) and pins (on the manifold) compensate for initial misalignment of the panel. Since the connectors on the manifolds are recessed, the connectors on the panel and manifold do not make contact until the panel is centered on the pins (that is, aligned) and pushed into the manifold. This approach to connector fine alignment may also benefit spacecraft alignment considerations for pointing accuracy.

The QIN resembles a standard bolt joint typically used in aerospace applications and therefore benefits from using a similar analysis. When the QIN is "torqued" to the designed value, it imparts elastic deformation in the nut, keeping the bolts from backing out. This is a significant issue for satellite structures where exotic methods are required to ensure a bolt does not back out.

A feasibility demonstration using a subscale prototype (a representative "corner" of three satellite panels assembled using the QIN fasteners, manifold and electrical connection) was performed to prove the rapid assembly concept. A scaled (three times smaller) demonstration model of the actual spacecraft bus (3' X 3' x 2') was designed and fabricated with the quick insertion fastening strategy. 
Table 1: Comparison of Standard Approach to Approach in Development

\begin{tabular}{|c|c|}
\hline \multicolumn{2}{|c|}{ Comparison of Standard Nut to Quick Insertion Nut (Fastening time alone) } \\
\hline Standard Nut & Quick Insertion Nut \\
\hline $\begin{array}{l}\text { - } \\
\text { - } \\
\text { Thread bolt onto nut by hand (to } \\
\text { prevent "cross threading” the bolt) } \\
\text { [TIME CONSUMPTION STEP - this } \\
\text { step alone is } 8 \text { to } 10 \text { times faster with } \\
\text { QIN] } \\
\text { - Using a wrench (or by hand) rotate bolt } \\
\text { until its head "bottoms out" against } \\
\text { mating part [TIME CONSUMPTION } \\
\text { STEP] } \\
\text { Using a torque wrench rotate bolt to } \\
\text { preload mating parts }\end{array}$ & 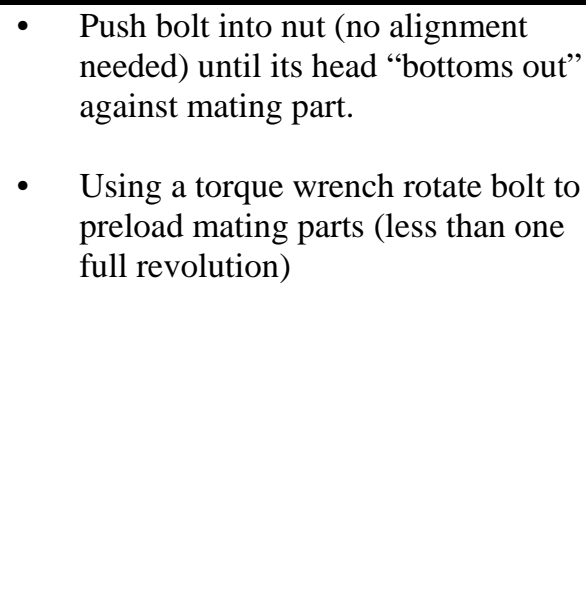 \\
\hline \multicolumn{2}{|c|}{$\begin{array}{l}\text { Other time-savings (not fastener-specific): } \\
\text { - } \quad \text { Electrical connection simultaneous instead of standard process of harness } \\
\text { routing and interconnect steps } \\
\text { - Thermal connection - will look at the benefits in the continuation of this } \\
\text { effort } \\
\text { - Potential to reduce or eliminate coupled loads analysis, random vibe, and } \\
\text { thermal vacuum testing and associated preparation time. To be considered in } \\
\text { detail in future work. }\end{array}$} \\
\hline
\end{tabular}

One corner of the prototype model consists of a base panel, two side panels, one vertical manifold and two horizontal manifolds, see Figure 5 and Figure 6. Integrated in the manifolds are the quick insertion nuts (Figure 7) and the electrical connectors. Integrating the electrical connector into the manifold eliminates the need for electrical "pigtails" on the panels and the manifolds. This also aids in the rapid assembly of the spacecraft bus by removing the need for manual connections of the electrical connectors (via electrical "pigtails") during assembly or the arduous processes associated with harnessing in traditional spacecraft AI\&T.
An electronic box containing a battery, LEDs and an on/off switch was wired and mounted on the base panel. The LED indicates electrical continuity across the connectors when a side panel is installed. Assembly of the demonstration model was completed and a video was made to document the assembly sequence of the panels. The demonstration video clearly shows how rapidly a panel can be installed, with simultaneous electrical connections, using the QIN fastening strategy. The video can be viewed at:

http://www.honeybeerobotics.com/168/Assembly4.mov 


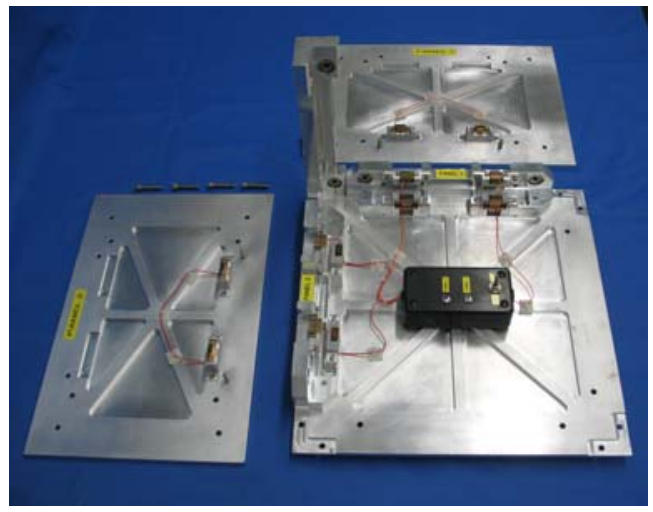

Figure 5: "Corner” of prototype model: base plate, manifolds and side panels.

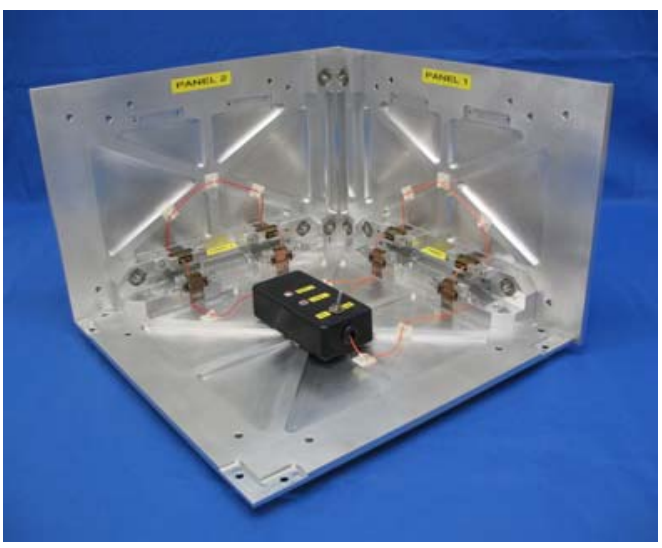

Figure 6: Assembled prototype model (note LEDs are "on" indicating continuity across panels).

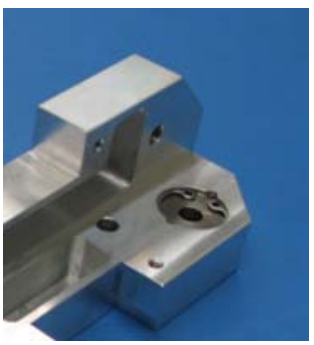

Figure 7: QIN assembled in Demo Model manifold.

\section{DETAIL DESIGN AND ANALYSES}

\section{Development of a Quick Removal Tool}

It is believed that the rapid disassembly of an assembled satellite structure could also be advantageous and therefore, of importance to RS' efforts. One of the advantages of the QIN is the fastener is rapidly inserted, with a translational motion, into the nut. Rapid disassembly of the panels from an integrated structure using standard tooling may be accomplished by removing the preload and using the quick-removal feature on the QIN or a modified version of it. Using the same features that allow for the fastener to be engaged rapidly, Honeybee Robotics has developed a concept for a tool (Figure 8) that would allow the fastener to be rapidly disengaged from the nut. In order for the fastener to be removed from the nut, the axial preload between the fastener and the nut has to be removed. In addition, the fastener has to be unscrewed about two turns (depending on the pitch of the fastener) to create a separation between the fastener's head and the clamped parts. Tabs in the tool move under the head of the fastener while a pin pushes the separated thread segments against the tapered washer in the QIN (a modification to the design of the threaded segments is needed to facilitate this action). With the threaded segments displaced outward (due to movement up the tapered washer), the fastener is free to ratchet over the threads of the nut in both directions. A linear pulling movement in the tool causes the tabs to pull the fastener out of the nut.

\section{Development of the Fastening Scheme of Satellite Bus}

Using the design of Spaceworks, Inc. panels and our manifold design concept, a preliminary concept of the satellite has been developed. In Spaceworks' design, all the side panels and the top/bottom decks are structurally identical. Each deck or panel is assembled from two identical panel halves, where the inside and outside surfaces of the panel assembly are identical. The key advantage of Spaceworks' panel design is the internal volume between the panels is used to contain the plugand-play electronics infrastructure. All the components and electronics are mounted to internal or external surfaces of the completed panels using a $5.0 \mathrm{~cm} \times 5.0 \mathrm{~cm}$ mounting hole pattern. The internal cutout of the panel is done in an iso-grid pattern.

The approach to rapid assembly of satellites involves integrating structural "manifolds" at the corners and along the top and bottom of the panels of the satellite. The manifolds at the corners (vertical manifolds) permit side panel-to-panel attachment while the manifolds along the top and bottom (horizontal manifolds) permit side panel to top/bottom panel attachment. The combination of vertical and horizontal manifolds creates a skeletal frame for the satellite, see Figure 9 \& Figure 11.

The vertical and horizontal manifolds are bolted together at the corners of the frame. The complete frame can be assembled without the panels and decks. A corner block on the vertical manifold aids fastening the horizontal manifolds of the frame. The corner block has a QIN to enable the same rapid assembly in the frame as the panel to manifold assembly. The corner blocks (two per vertical manifold) are expected to be pre-assembled (using standard fasteners) onto the vertical manifolds prior to assembly of the entire bus. 


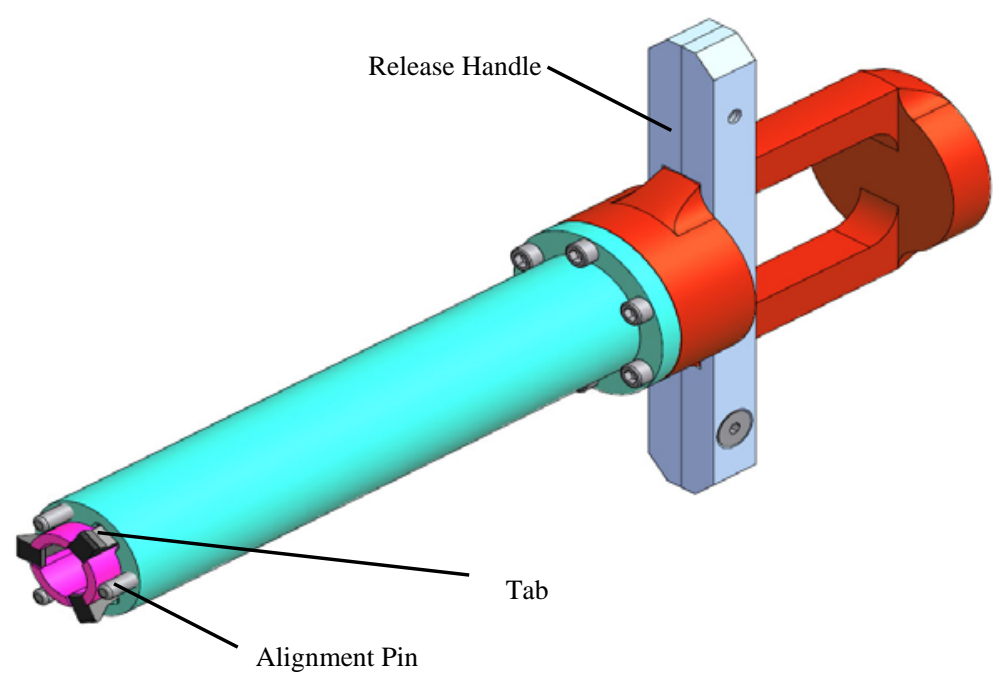

Figure 8: Quick Removal Tool Concept for the QIN

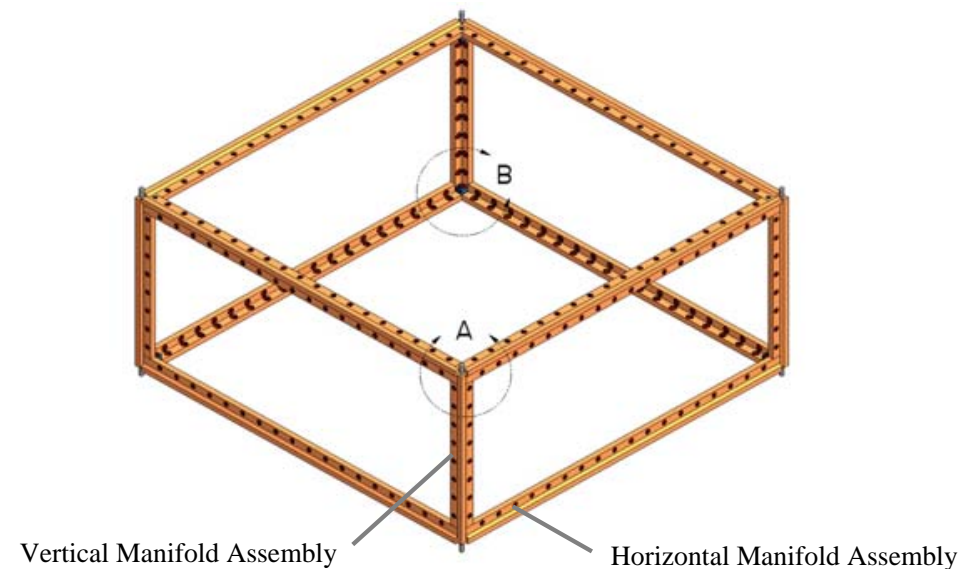

Figure 9: Skeletal Attachment Frame for Satellite's panels

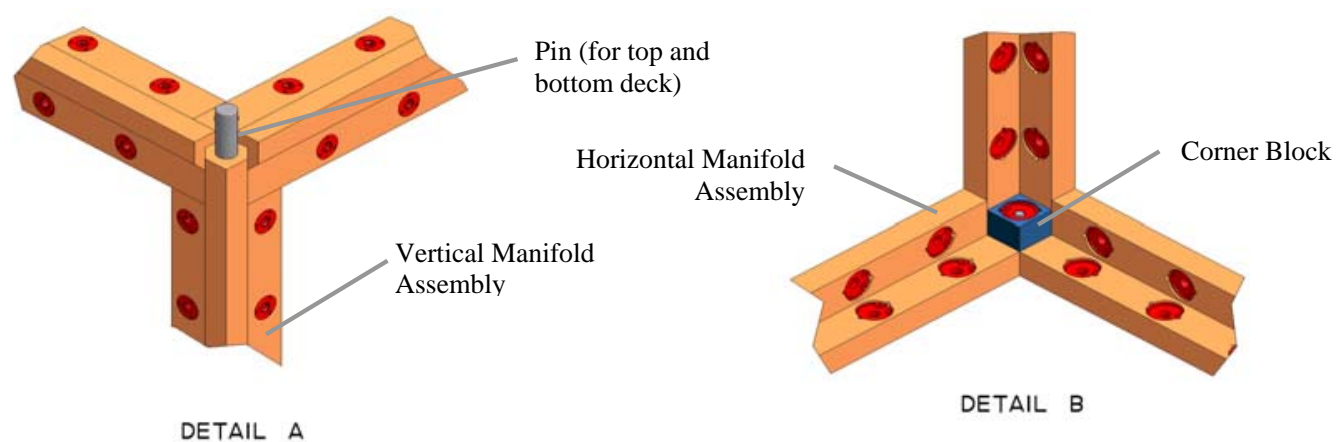

Figure 10: Corner detail of Satellite's frame 


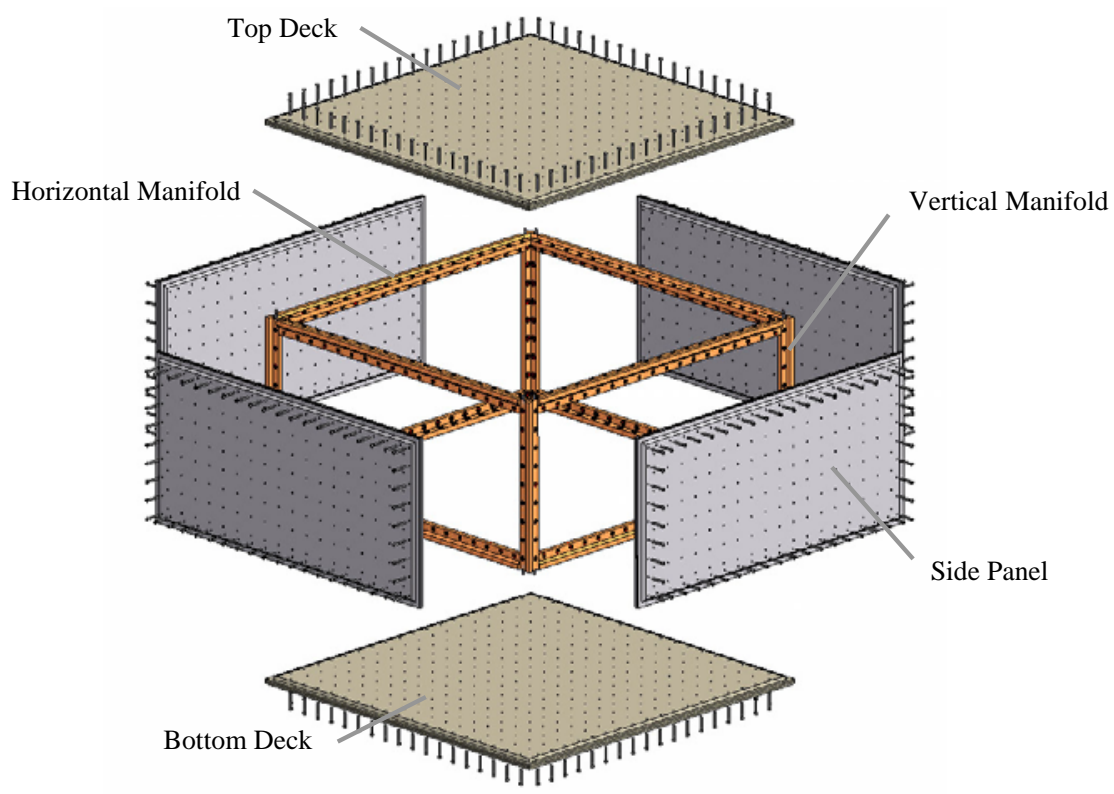

Figure 11: Exploded satellite bus structure showing the manifold frame and panels.

If needed, heat pipes can be integrated into the manifolds to aid the thermal management and control of the satellite. Integrated and rotationally fixed within the manifolds are the quick insertion nuts for mounting the panels. The manifold structure is a simple shape that can be easily extruded during the manufacturing process. The counter-bored holes for the QINs would then be machined into the extruded manifold structure.

The same fundamental design concept can be used to construct different satellite geometrical configurations (i.e., rectangular, cubic or hexagonal). The only major change in the design would be the length of the manifolds and the included angle of the vertical manifolds. For example, a 90-degree included angle vertical manifold creates a cubic or rectangular shaped satellite while a 120-degree included angle manifold creates a hexagonal shaped satellite.

To further the manifold attachment concept, modal Finite Element Analysis (FEA) was performed to determine the natural frequency of this satellite construction. The size requirement of the satellite model was taken to be a square configuration of 1 meter by 1 meter by $1 / 2$ meter high. The side panel assemblies (based on the SpaceWorks, Inc. design) is comprised of a "clam shell" design of two individual side panels measuring $0.9492 \mathrm{~m}$ by $0.4492 \mathrm{~m}$ by $0.0127 \mathrm{~m}$ thick. The top and bottom deck assemblies are also comprised of a "clam shell" design of individual panels measuring $0.9492 \mathrm{~m}$ by $0.9492 \mathrm{~m}$ by $0.0127 \mathrm{~m}$ thick. The "hollowed out clam shell pocket" in a panel assembly is created from the two panel halves with material removed from the center. An iso-grid rib pattern is created in the center of the panels during material removal so as to retain the structural rigidity of the panels.

In order to perform the FEA modal analysis, it was first necessary to determine whether to perform threedimensional modeling or a simplified model representation of the satellite. Accurate modal analysis using 3-dimensional elements requires the mesh size to be the smaller than the smallest feature length in the part. If larger mesh sizes are used with 3-dimensional elements, then the analysis produces results that are much higher than the true natural frequency of the part. Therefore, creating a meshed model with $3 \mathrm{D}$ elements can result in a large number of elements which can be time consuming and requires greater computing power.

One way to decrease the number of elements in the model is to create parts using elements that are 2-dimensional such as plate and beam elements. A simplified representation of a panel was created using 2-D and 3-D features modeled in the FEA software. In the FEA software, the panel flat surface was modeled as plate elements, while the rib pattern was modeled as beam elements. Brick elements were used to create the 3dimensional frame of the panel.

The figures below show the simplified CAD and $3 \mathrm{D}$ visualization of the panel in the finite element software. 


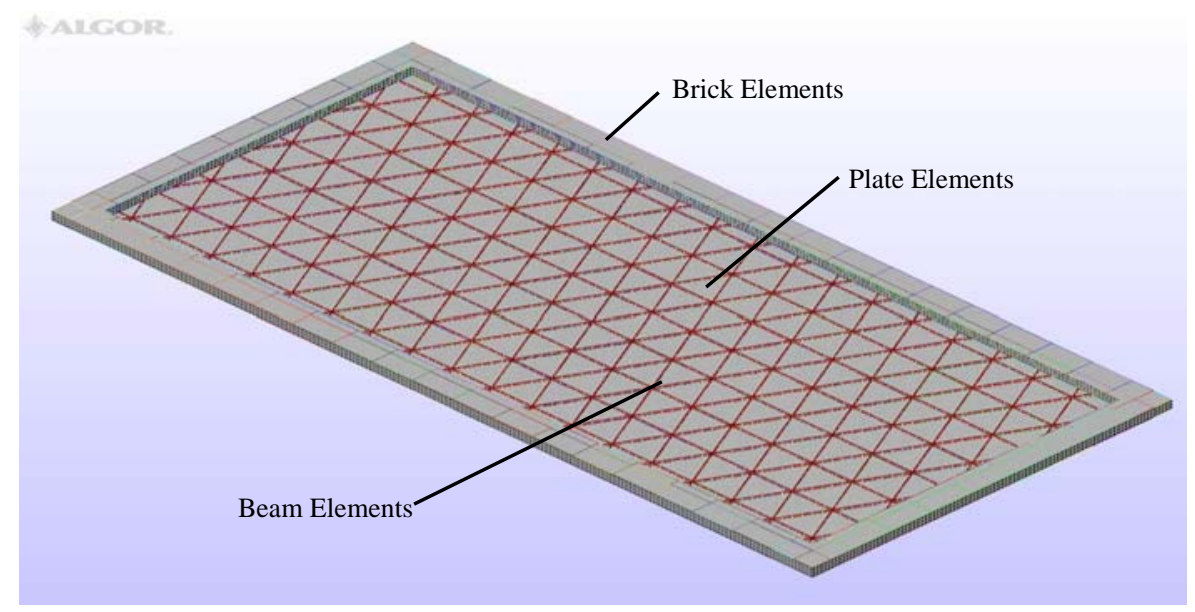

Figure 12: Simplified Panel Representation in FEA software

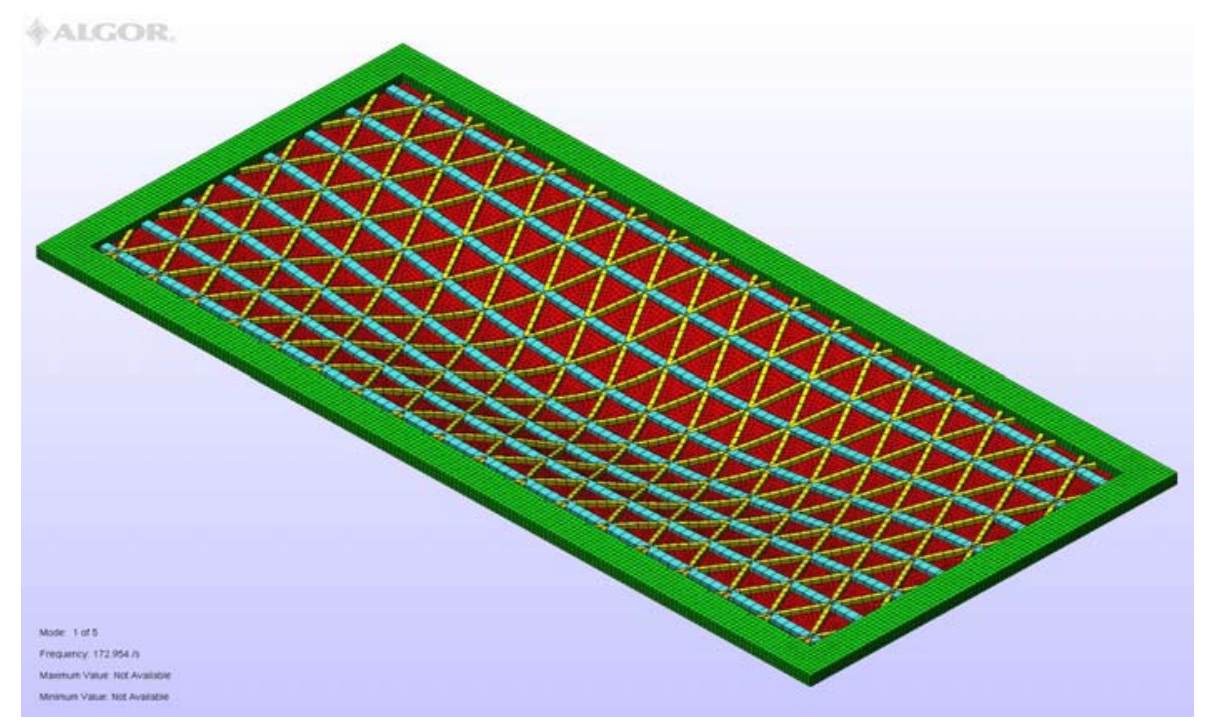

Figure 13: 3-dimensional display of simplified panel representation in FEA software

A model of the complete satellite bus was created using the simplified FEA panel model of brick, plate, and beam elements. The manifold frame was modeled as brick elements. The total mass of the complete satellite bus, before optimization, was 121.1 $\mathrm{kg}$. The mass requirement of the satellite bus, $200 \mathrm{~kg}$ located at its center, meant that additional mass had to be added to the model. A simple way to add mass to the model is to attach a point mass via rigid beams or springs. An additional mass of $9.8625 \mathrm{~kg}$ was added to the side panel assemblies and an additional mass of $19.725 \mathrm{~kg}$ was added to the top and bottom deck assemblies. The satellite bus bottom deck was fixed at 60 locations around a bolt circle representing the Planetary System Corporation's Lightband Separation System.

A payload mass of $200 \mathrm{~kg}$ located $0.6096 \mathrm{~m}$ above the satellite was attached to the top deck using the same bolt pattern of the Lightband Separation System of the bottom deck. A point mass was positioned $0.6096 \mathrm{~m}$ above the satellite and attached to the 60 nodes on the ring using rigid elements with a defined stiffness. The setup of this FEA model is shown in the Figure 14 below. The calculated frequency of the satellite bus is listed in Table 2 below, while the first mode shape is shown in Figure 15 \& Figure 16. 
Table 2: Modal frequencies of Satellite Bus Structure

\begin{tabular}{|c|c|}
\hline Mode & Frequency $[\mathrm{Hz}]$ \\
\hline 1 & 29.74 \\
\hline 2 & 35.03 \\
\hline 3 & 35.66 \\
\hline 4 & 36.78 \\
\hline 5 & 64.89 \\
\hline 6 & 70.63 \\
\hline 7 & 71.38 \\
\hline 8 & 72.09 \\
\hline 9 & 72.40 \\
\hline 10 & 156.11 \\
\hline
\end{tabular}

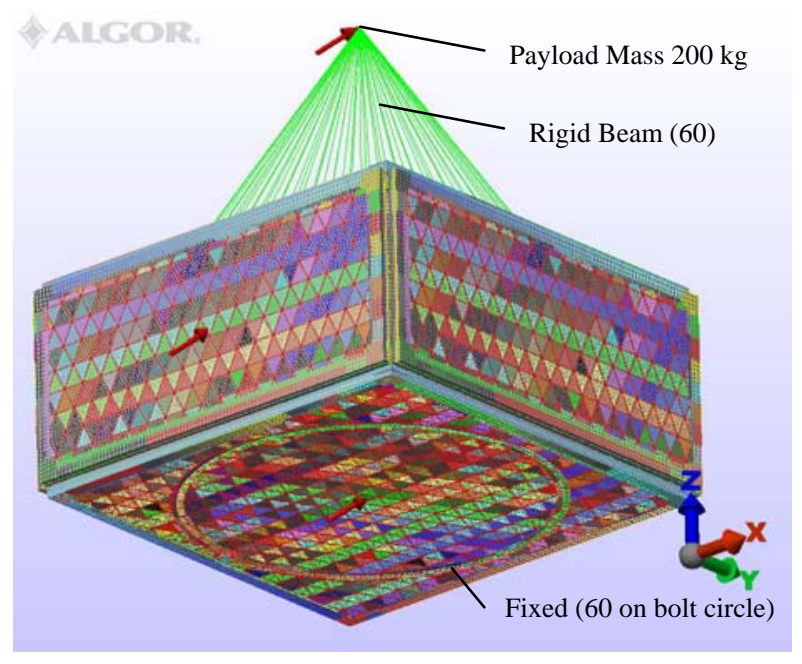

Figure 14: Finite Element Analysis Constraints

The analysis presented above is preliminary but helps to further optimize the bus' design. Some additional considerations for redesign and optimization are:

- Add stand-offs between the middle of the panel assembly to better tie the panel halves together (currently only the frame at the end are tied together onto the manifold)

- Increase the iso-rib pattern spacing

- Increase the thickness of the plate section on the larger panels (the top and bottom deck panels) to increase their stiffness

- Use mass-less rigid beams to tie the payload and additional satellite mass to the panels

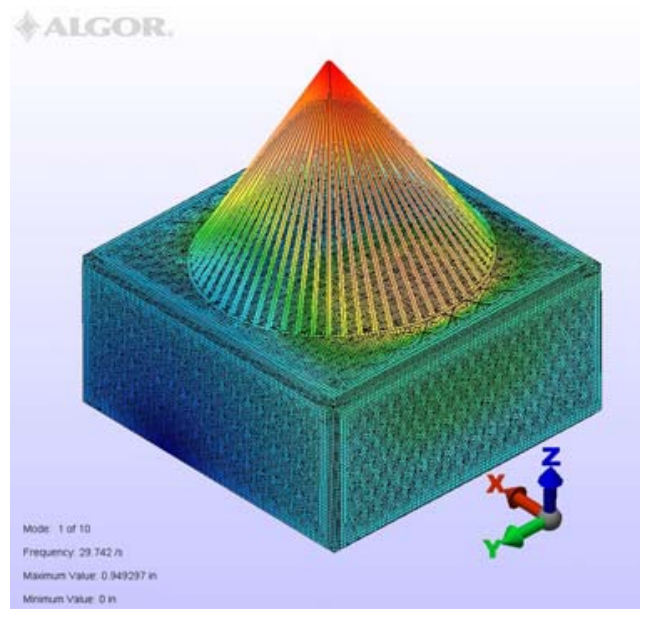

Figure 15: First Modal Frequency of Satellite

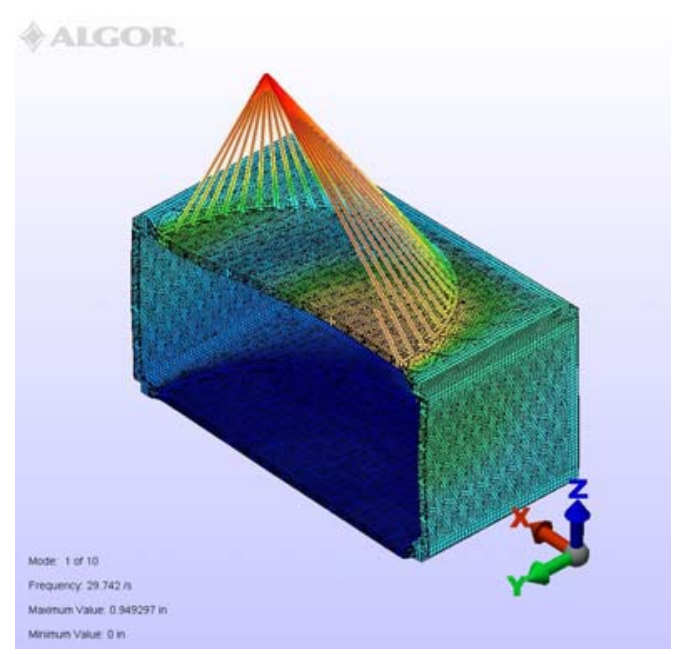

Figure 16: First Modal Frequency (cut away view)

\section{Finite Element Analysis of a Quick Insertion Nut}

Frequently asked questions about the QIN are: how does it behave in a bolted joint and can classical analyses for a standard nut and joint be used? It is believed that the QIN is not different (except for the quick installation feature) from a standard nut, and that it could, therefore, be analyzed using the same classical methods and techniques. Honeybee Robotics has always maintain that the QIN should perform better than a standard nut because of the tapered or conical hole the threaded segments seat into as the fastener is preloaded. This seating due to the tapered contact between the segments and the housing result in better load distribution on the 
threads as the fastener/nut is preloaded. Finite Element Analysis (FEA) was performed on the Quick Insertion Nut to determine if it behaves like a standard nut. A 3-dimensional model of the QIN and the bolt was created (inducing the threads) in Algor's FEA software. The threads were modeled using the standard Unified Screw Thread notation and the basic dimensions of the thread size in references such as the Machining Handbook. To simplify the analyses, the thread contact between the bolt and nut were considered to be a bonded contact (in reality this is not true, as the threads can slide relative to each other when loaded). In bonded contact, the two surfaces will be in perfect contact throughout the analysis and the loads are transmitted from one part to the adjacent part. In the analysis, when a node on one surface deflects, the node on the adjoining surface will deflect the same amount in the same direction. Considering surface contact between the threads is a more complicated analysis that will be performed later. The FEA model (Figure 17) was setup similar to the test fixtures used in the National Aerospace Standard Fastener testing method NASM 1312-8. In this test, two tension fixture plates are used to load the fastener and nut joint. The head of the fastener preloads against one of the tension fixture plates (ground), while the nut is loaded by the other fixture plate.

A total axial force of $25221.42 \mathrm{~N}$ (5670 lbs) was applied to the 4 corners of the tension plate against the nut. A large force was selected to ensure failure of the thread of the quick insertion nut. In the FEA software, the force was applied in increments over a period of time. Only the linear section of the materials modulus of elasticity was used in the analysis, there was no accounting for strain hardening. As such, the stress result will remain linear after the yield point of the material i.e. the result will be based on the linear slope of the stress-strain curve. This is acceptable because failure for this analysis was defined as any stress greater than the yield strength of the material.

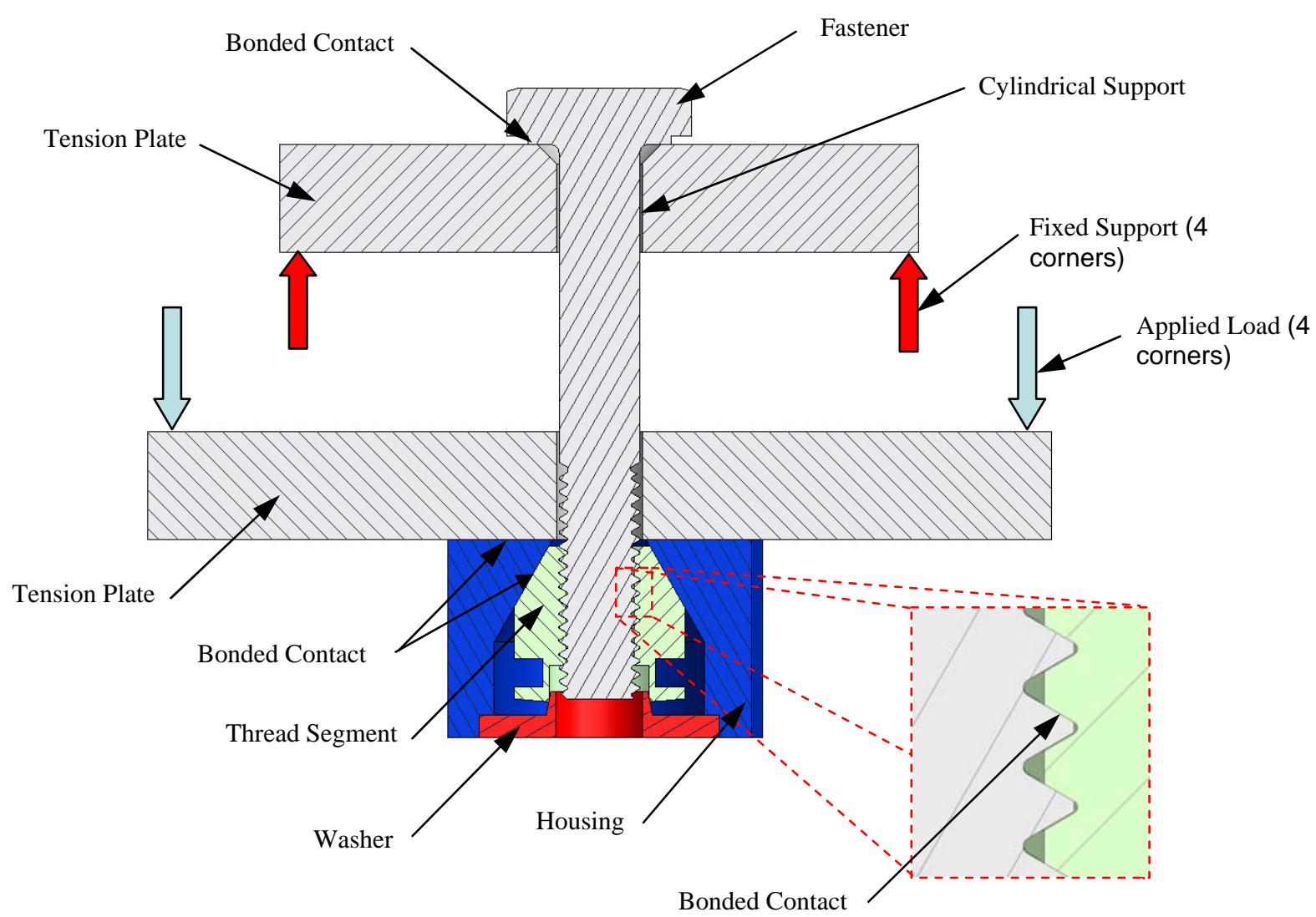

Figure 17: Finite Element Setup of QIN model 


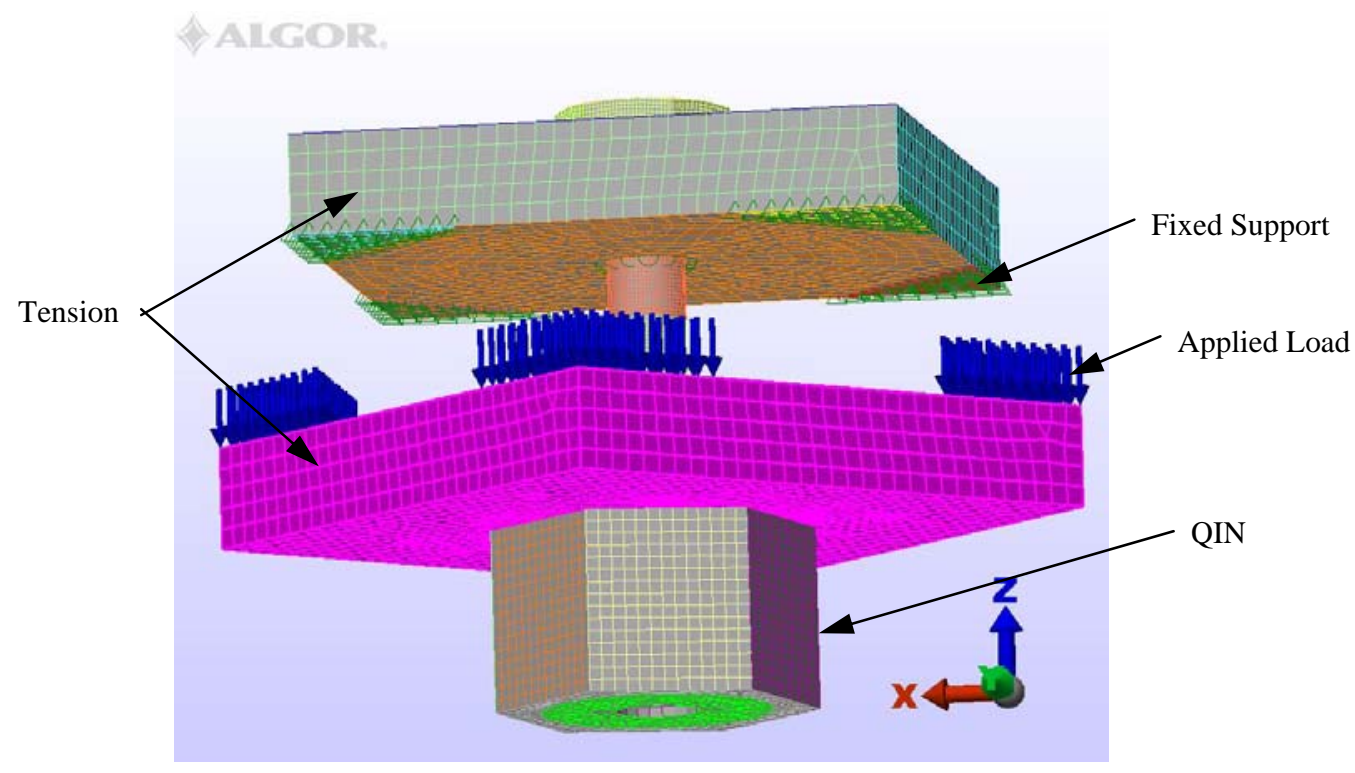

Figure 18: Meshed FEA model.

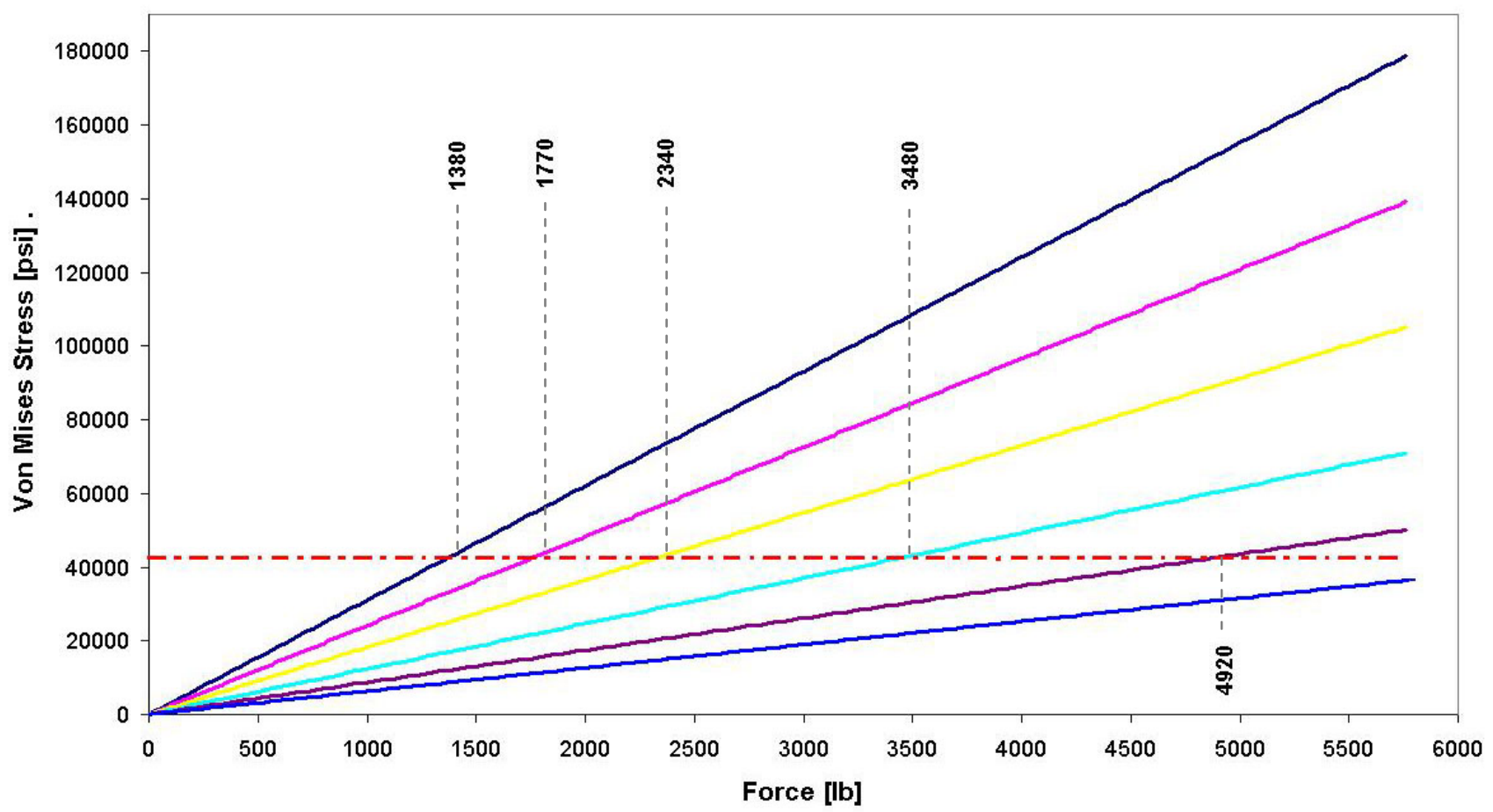

- Thread \#1 $\longrightarrow$ Thread \#2 $\quad$ Thread \#3 - Thread \#4 - Thread \#5 - Thread \#6 - - Thread Yield Strength

Figure 19: Average Von Mises Stress of the Thread in the QIN 
The graph (Figure 19) shows the average von mises stress of the threads in the QIN. Thread \#1 is one complete revolution on the thread helix (the pitch of the nut). The load required to fail the threads in the nut increases as the number of threads increase in the nut. This is consistent with the phenomenon that is seen in a standard nut, in which the load bearing capacity increases with the number of threads. In fact, in a fastener joint (bolt and nut) only the first few threads (4-5) carry the majority of the tensile load.

This trend was seen (Figure 19 ) and illustrated by the finite element analysis (Figure 20 ) of the Quick Insertion Nut. The analysis shows that the QIN model does behave like a conventional nut in that the first few (5-6) threads carry most of the load. Once the first thread fails, the load is redistributed over the next five threads. This process will continue until all the threads fail.

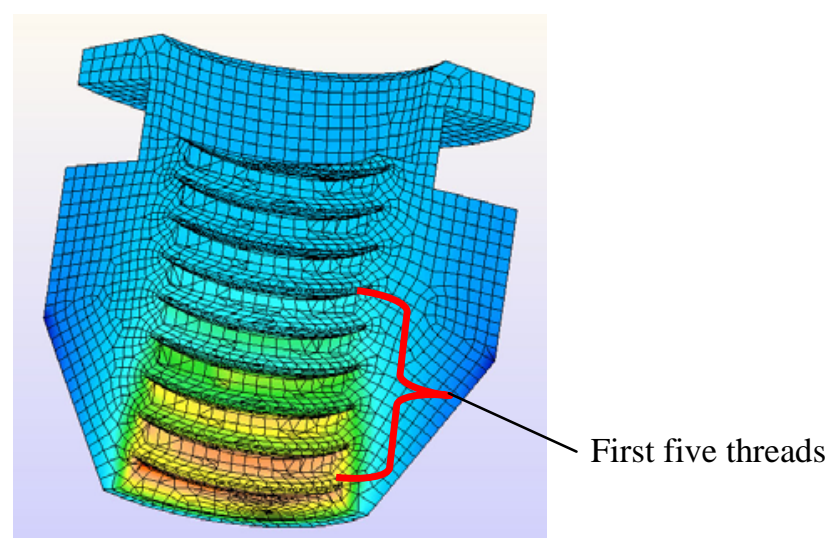

Figure 20: First few threads loaded in QIN

\section{CONTINUATION OF THIS EFFORT}

The following continuation of this effort is planned in order to develop the rapid assembly concept described in this paper to the point at which it is ready for use on satellite structures.

\section{- Mechanical and Physical Tests}

Tensile Strength - This test method determines the tensile strength of externally and internally threaded fasteners. Tensile strength testing for the QIN shall be performed according to the latest revision of NASM 1312-8 “Tensile Strength” (formerly MID-STD-1312-8A) or ASTM F606 "Standard test method for determining mechanical properties for external and internal threaded fasteners.”
Torque Tension - This test method determines the room temperature torque-tension relationship for threaded fasteners. Torque tension for the QIN shall be performed according to the latest revision of NASM1312-15 “Torque Tension.”

Torque-Angle and Torque to Yield Test in Satellite's panel representation - This test method determines the torque needed to reach the yield strength of the QIN. Tests can be preformed on the actual assembly to obtain the torque angle graphs (Number of turn vs. Torque).

Failure Test - This test method determines the torque-angle characteristics of the QIN by tightening it to failure. Tests can be preformed on the actual assembly or test fixture to allow torque-force characteristics to be established.

\section{- Vibration Tests}

Standard Sine Sweep - The QIN shall be vibration tested based on standard sine sweep.

Military Specification Vibration - The QIN shall be vibration tested based on military specification vibration levels

\section{- System satellite bus level testing}

- Rapid assembly of a Satellite Bus Mockup using the QINs

- Thermal-vacuum and vibration testing of Satellite Bus Mockup with QINs

\section{References}

1. Lyke, J., et al., "Space Plug-and-Play Avionics," AIAA 3rd Responsive Space Conf, Paper No. RS3-2005-5001, Los Angeles, CA, 25 - 28 Apr 2005.

2. Lee, Douglas E., "Space Reform," Air and Space Power Journal, Summer 2004.

3. Arritt, B., Roopnarine, et al, "Development of a Satellite Structural Architecture for Operationally Responsive Space,” abstract for 2008 Structures, Structural Dynamics, and Materials (SDM) Conference

4. Minotaur Orbital Sciences Corporation, “Minotaur I User's Guide," Release 2.1, Jan 2006. 
5. Arritt, B., et al, "Responsive Satellites and the Need for Structural Health Monitoring," SPIE Smart Structures and Materials and Nondestructive Evaluation and Health Monitoring, March 2007

6. http://www.responsivespace.com/Papers/RS 5\%5CSESSION\%20PAPERS\%5CSESSIO N\%205\%5C5003_SARAFIN\%5C5003P.PD $\mathrm{F}$

7. Wegner, P., Kiziah, R., "Pulling the Pieces Together at AFRL," AIAA 4th Responsive Space Conf, No. RS4-2006-4002, Los Angeles, CA, 24 - 27 Apr 2006.

8. Williams, A., Palo, S., "Issues and Implications of the Thermal Control Systems on the 'Six Day Spacecraft'," AIAA 4rd Responsive Space Conference, Paper No. RS4-2006-6001, Los Angeles, CA, 24 - 27 Apr 2006

9. Roopnarine, inventor; Honeybee Robotics Inc., assignee. Quick insertion and removal fastener. US patent 6,712,574 B1. 2004 Mar 30 